\title{
Hallazgos en la endoscopia digestiva alta en niños con talla baja
}

\section{Findings of upper gastrointestinal endoscopy in children with short stature}

\author{
Julio César Castro-Murillo, ${ }^{1}$ (D) Carlos Alberto Velasco-Benítez, ${ }^{2 *}$ (i] 0tto Gerardo Calderón-Guerrero. ${ }^{3}$ (ib
}

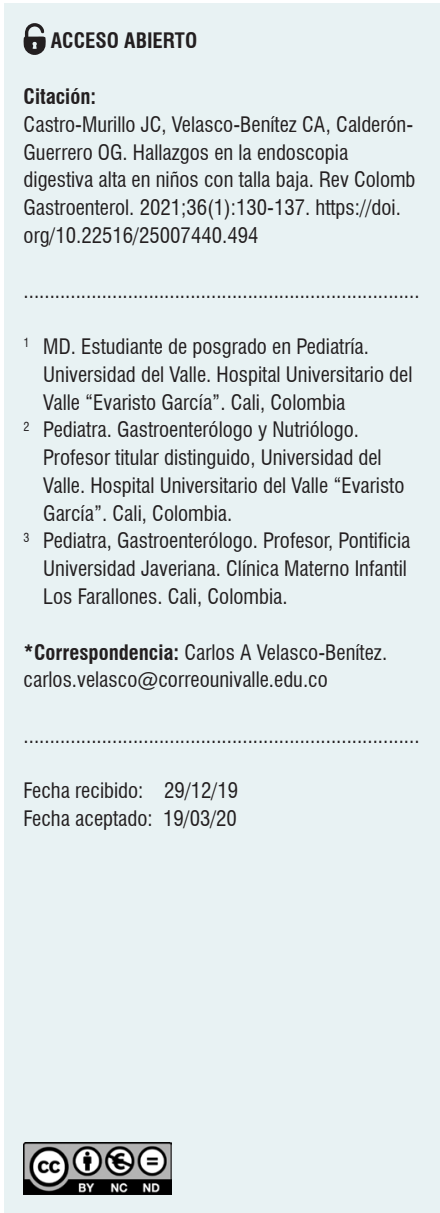

\begin{abstract}
Resumen
Introducción: en el estudio de la baja talla de origen digestivo en niños no hay un patrón de paraclínicos preestablecido; sin embargo, la endoscopia de vías digestivas puede ser una herramienta útil para tal fin. Objetivo: reportar una serie de casos de niños con diagnóstico de baja talla a quienes se les indicó una endoscopia de vías digestivas altas como parte de su estudio. Reporte de casos: se incluyeron 15 niños entre los 2 y 16 años de edad, 53,3 \% niñas, $26,7 \%$ desnutridos según el índice de masa corporal y la talla para la edad, $66,7 \%$ con baja talla grave y $33,3 \%$ con baja talla moderada. El $53,3 \%$ presentó dolor abdominal, el $46,7 \%$ no tuvo ganancia de peso, el $26,7 \%$ tuvo inapetencia y el $13,3 \%$ tuvo vómito, entre otros. Entre el $40,0 \%$ y el $93,4 \%$ presentaron macro- o microscópicamente esofagitis, gastritis y duodenitis. Los hallazgos microscópicos más importantes fueron duodenitis crónica con giardiasis, úlceras duodenales, hiperplasia nodular linfoide duodenal, Helicobacter pylori y duodenitis crónica eosinofilica. Conclusiones: a pesar de que la endoscopia de vías digestivas es un método poco utilizado y no bien descrito en el estudio de niños con baja talla, este reporte de casos describe organicidad en un $80,0 \%$ de los niños analizados.
\end{abstract}

\section{Palabras clave}

Insuficiencia de crecimiento, niños, endoscopia.

\begin{abstract}
Introduction: The study of short stature of digestive origin in children shows no pre-established laboratory patterns. However, endoscopy of the digestive tract may be a useful tool for this purpose. Objective: To report a series of cases of children with a diagnosis of short stature who underwent upper digestive tract endoscopy as part of their study. Case report: 15 children between the ages of 2 and 16 years were included; $53.3 \%$ were girls. $26.7 \%$ presented with malnutrition according to their body mass index and height-for-age, $66.7 \%$ had short stature, and $33.3 \%$ moderate short stature. Abdominal pain was reported in $53.3 \%$ of the cases, and no weight gain in $46.7 \%$. Other symptoms were lack of appetite in $26.7 \%$, vomiting in $13.3 \%$, among others. Between $40 \%$ and $93.4 \%$ of the children presented macro and/or microscopic esophagitis, gastritis, and duodenitis. The most important microscopic findings were chronic duodenitis with giardiasis, duodenal ulcers, duodenal nodular lymphoid hyperplasia, Helicobacter pylori, and chronic eosinophilic duodenitis. Conclusions: Although endoscopy of the digestive tract is a method barely used and not well described in the study of children with short stature, this case report describes organicity in $80 \%$ of the children analyzed.
\end{abstract}

\author{
Keywords \\ Growth failure, Children, Endoscopy.
}




\section{INTRODUCCIÓN}

La baja talla en niños se determina luego de una valoración nutricional en la que se observa que no tienen ganancia de talla acorde con su crecimiento (1). La baja talla es frecuente y ocurre cuando la ingesta calórica es insuficiente para mantener el crecimiento (2). Las diferentes causas de baja talla pueden ser psicosociales, orgánicas y no orgánicas; estas últimas en una minoría de casos, y su principal signo de alarma es la dificultad para tener o mantener un peso adecuado (2).

La baja talla es reversible en la mayoría de los casos por medio de intervención nutricional; de lo contrario, su persistencia la puede convertir en una enfermedad orgánica subyacente que amerita la toma de pruebas de laboratorio, imagenológicas y otras, como la endoscopia de vías digestivas (EVD) en busca de su etiología (2). Desde el punto de vista digestivo, puede ser secundaria a enfermedad por reflujo gastroesofágico, a enfermedad celíaca y a otras alteraciones crónicas $(3,4)$.

Para el presente reporte de casos se tuvo en cuenta la clasificación de la Organización Mundial de la Salud (OMS) (5), que incluye a los niños con $>-2$ desviaciones estándar (DE) de la talla/edad (TE). Dado que en esta definición el peso/talla y el índice de masa corporal (IMC) pueden estar normales, la baja talla puede estar relacionada con procesos crónicos a largo plazo y de condición orgánica.

No hay un estándar de pruebas complementarias específicas; sin embargo, la indicación de paraclínicos por parte de un grupo multidisciplinario son primordiales en el manejo inicial de los niños con baja talla, y la EVD es una herramienta útil para descartar causas digestivas, lo que permite la identificación macro- y microscópica que evidencie una alteración orgánica como causa de la baja talla $(2,3,6)$.
El objetivo de este escrito es reportar una serie de casos de niños entre los 2 y 16 años de edad con diagnóstico de baja talla de una unidad de endoscopia pediátrica (UEP) de Cali, Colombia, según la definición de la OMS, y a quienes dentro de sus estudios se les indicó una endoscopia de vías digestivas altas (EVDA).

\section{REPORTE DE CASOS}

\section{Características generales}

Se incluyeron 15 niños de 7,1 $\pm 4,9$ años (rango $=2-16$ años): 46,7 \% preescolares (2-5 años), 40,0 \% escolares (6-12 años) y 13,3 \% adolescentes (13-16 años); 53,3 \% de sexo femenino que asistieron a una UEP de Cali, Colombia. Los promedios $\pm \mathrm{DE}$ y rangos fueron los siguientes: peso $=$ $16,4 \pm 9,3 \mathrm{~kg}($ rango $=7,8-39,9 \mathrm{~kg})$, talla $=100,6 \pm 24,6 \mathrm{~cm}$ $($ rango $=74-148 \mathrm{~cm})$, IMC según la OMS $=-0,8 \pm 1,2 \mathrm{DE}$ $($ rango $=-3,1$ y $+1,4 \mathrm{DE})$ y TE $=-3,9 \pm 1,3 \mathrm{DE}($ rango $=-6,4$ y -2,0 DE); tenía desnutrición según el IMC para la OMS el $26,7 \%$ y, según TE para la OMS, el 66,7 \% presentaba baja talla grave y el $33,3 \%$, baja talla moderada.

\section{Sintomatología}

Se presentaron síntomas como dolor abdominal en el 53,3\%, no ganancia de peso en el $46,7 \%$, inapetencia en el $26,7 \%$, vómito en el $13,3 \%$ y diarrea y distensión abdominal en el $6,7 \%$, respectivamente (Tabla $\mathbf{1}$ ).

\section{Hallazgos macro- y microscópicos}

Los principales hallazgos en la EVDA y en las biopsias fueron, en orden de frecuencia, esofagitis, gastritis y duodenitis, entre un 40,0 \% y un 93,4 \% (Tabla 2).

Tabla 1. Sintomatología dependiente de los hallazgos microscópicos de 15 niños con baja talla de una unidad de endoscopia pediátrica de Cali, Colombia

\begin{tabular}{|c|c|c|c|c|c|c|c|c|c|}
\hline & \multicolumn{2}{|c|}{ Esófago $(n, \%)$} & \multirow[t]{2}{*}{$p$} & \multicolumn{2}{|c|}{ Estómago (n, \%) } & \multirow[t]{2}{*}{$p$} & \multicolumn{2}{|c|}{ Duodeno (n, \%) } & \multirow[t]{2}{*}{$p$} \\
\hline & Normal & Inflamado & & Normal & Inflamado & & Normal & Inflamado & \\
\hline Dolor abdominal & $4(26,7)$ & $4(26,7)$ & 0,659 & $4(26,7)$ & $4(26,7)$ & 0,659 & $4(26,7)$ & $4(26,7)$ & 0,659 \\
\hline No ganancia de peso & $1(6,7)$ & $6(40,0)$ & 0,040 & $5(33,3)$ & $2(13,3)$ & 0,195 & $3(20,0)$ & $4(26,7)$ & 0,599 \\
\hline Inapetencia & $0(0,0)$ & $4(26,7)$ & 0,050 & $2(13,3)$ & $2(13,3)$ & 0,701 & $1(6,7)$ & $3(20,0)$ & 0,299 \\
\hline Vómito & $0(0,0)$ & $2(13,3)$ & 0,241 & $1(6,7)$ & $1(6,7)$ & 0,759 & $1(6,7)$ & $1(6,7)$ & 0,759 \\
\hline Diarrea & $1(6,7)$ & $0(0,0)$ & 0,500 & $0(0,0)$ & $1(6,7)$ & 0,500 & $1(6,7)$ & $0(0,0)$ & 0,500 \\
\hline Distensión abdominal & $1(6,7)$ & $0(0,0)$ & 0,500 & $0(0,0)$ & $1(6,7)$ & 0,500 & $1(6,7)$ & $0(0,0)$ & 0,500 \\
\hline
\end{tabular}


Tabla 2. Hallazgos macro- y microscópicos en 15 niños con baja talla de una unidad de endoscopia pediátrica de Cali, Colombia

\begin{tabular}{|lccc|}
\cline { 1 - 2 } \multicolumn{1}{c}{ Hallazgos } & Macroscópicos & Microscópicos & $\boldsymbol{p}$ \\
\cline { 2 - 3 } & $\mathbf{n ( \% )}$ & $\mathbf{n}(\%)$ & \\
\hline Esofagitis & $14(93,4)$ & $11(73,4)$ & 0,165 \\
Gastritis & $13(86,7)$ & $7(46,7)$ & 0,025 \\
Duodenitis & $12(80,0)$ & $6(40,0)$ & 0,030 \\
Esofagitis, gastritis, & $9(60,0)$ & $3(20,0)$ & 0,030 \\
duodenitis & & & \\
\hline
\end{tabular}

Se identificaron otros hallazgos de importancia como duodenitis crónica con giardiasis (Figura 1), úlceras duodenales (Figura 2), hiperplasia nodular linfoide duodenal, Helicobacter pylori y duodenitis crónica eosinofílica (Tabla 3).

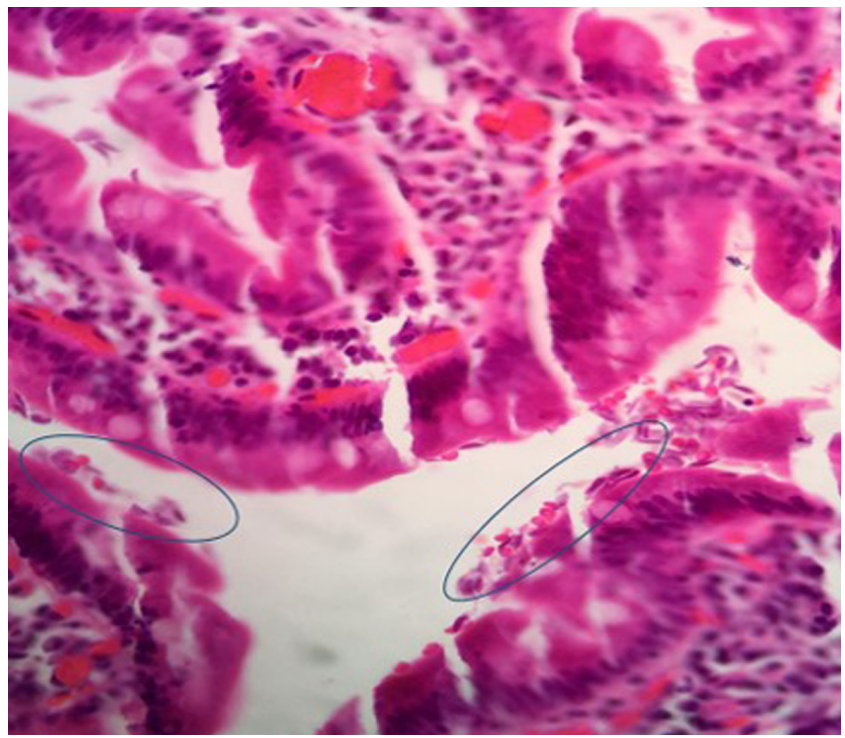

Figura 1. Niña de 2 años de edad con duodenitis crónica y giardiasis. Cortesía de Ramiro Pineda. Clínica Materno Infantil Los Farallones. Cali, Colombia.

\section{Giardiasis, H. pylori y baja talla}

Para el análisis de la asociación entre Giardia lamblia, $H$. pylori y baja talla (Tablas 4 y 5 ), se realizó la búsqueda de artículos originales en PubMed con las palabras en español, "malnutrición" y "niños"; en inglés, "malnutrition" y "children"; y en portugués, "desnutrição" y "criancas", entre el 1 de enero de 2000 y el 31 de octubre de 2019. La búsqueda solo incluyó artículos originales que describieran resultados de investigación. Se tuvo en cuenta principalmente la prevalencia para giardiasis, baja talla y la posible asociación entre giardiasis y baja talla. No se incluyeron las publicaciones duplicadas determinadas por similares autores, tamaños de muestra ni país.

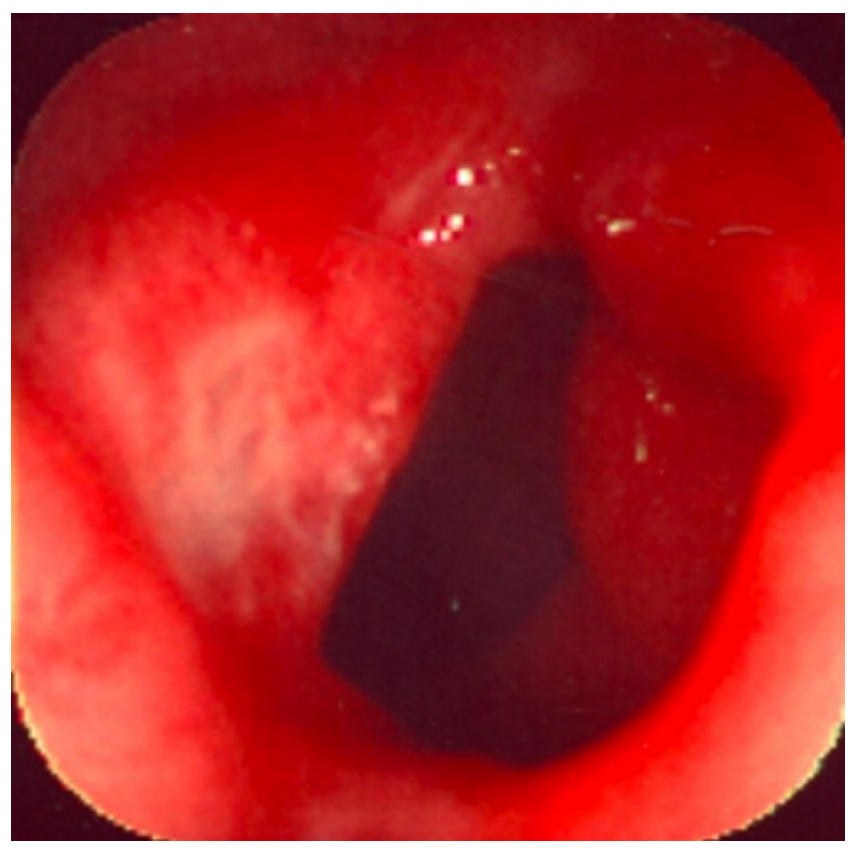

Figura 2. Niña de 3 años de edad con úlceras duodenales.

\section{DISCUSIÓN}

A pesar de que la EVD está indicada para para el estudio de los niños con baja talla (6), sigue siendo un método poco utilizado y no ha sido bien descrito; como lo reportaron Larson y colaboradores (28), quienes encontraron que la EVD es más útil que la imagenología o los paraclínicos en niños con baja talla y reportaron organicidad en un 16,7 \%; sin embargo, solo fue indicada en el $10 \%$ de los niños estudiados con baja talla.

12 de nuestros 15 niños estudiados $(80,0 \%)$ tuvieron mínimo una alteración a nivel del esófago, estómago o duodeno; datos diferentes a lo reportado por Larson y colaboradores (28), cuyos niños remitidos para baja talla a una clínica de gastroenterología pediátrica terciaria presentaron en su mayoría causas no orgánicas para baja talla, a pesar de que el $66,3 \%$ de ellos requirió de una prueba diagnóstica para evaluar la baja talla. El 40,0 \% de nuestros niños presentó duodenitis a la histopatología, datos similares a los de El Mouzan y colaboradores (29), quienes estudiaron a 116 niños con baja talla, se les tomó biopsia duodenal y reportaron en 65 de ellos anormalidad (44,0 \%), en la mitad de ellos atrofia vellosa y en la otra mitad, duodenitis crónica inespecífica.

Dos de nuestros pacientes presentaron baja talla grave con duodenitis crónica y giardiasis. Los estudios son controversiales en cuanto a la posible asociación de la giardiasis 
Tabla 3. Correlación clínico-patológica en 15 niños con baja talla de una unidad de endoscopia pediátrica de Cali, Colombia

\begin{tabular}{lclll} 
Sexo & Edad (años) & Diagnóstico nutricional & \multicolumn{1}{c|}{ Síntomas } & \multicolumn{1}{c}{ Hallazgos microscópicos } \\
\hline Femenino & 2 & Baja talla grave & Inapetencia & $\begin{array}{l}\text { Duodenitis crónica } \\
\text { Giardiasis }\end{array}$ \\
\hline Femenino & 2 & Baja talla grave & $\begin{array}{l}\text { Inapetencia } \\
\text { No ganancia de peso }\end{array}$ & $\begin{array}{l}\text { Duodenitis crónica } \\
\text { Giardiasis }\end{array}$ \\
\hline Masculino & 3 & Baja talla moderada & Vómito & Úlcera aguda prepilórica \\
\hline Femenino & 2 & Baja talla grave & Dolor abdominal & H. pylori \\
\hline Femenino & 2 & Baja talla moderada & No ganancia de peso & Hiperplasia nodular linfoide duodenal \\
\hline Masculino & 16 & Baja talla grave & No ganancia de peso & Duodenitis crónica eosinofílica
\end{tabular}

Tabla 4. Giardiasis y baja talla

\begin{tabular}{|c|c|c|c|c|c|c|c|}
\hline Autor & Año & País & $\mathbf{n}$ & $\begin{array}{l}\text { Edad } \\
\text { (años) }\end{array}$ & $\begin{array}{c}\text { G. lamblia } \\
(\%)\end{array}$ & $\begin{array}{l}\text { Falla para crecer } \\
\qquad(\%)\end{array}$ & Asociación \\
\hline Ihejirika (7) & 2019 & Nigeria & 300 & $5-13$ & 2,7 & 26,0 & No \\
\hline Lehto (8) & 2019 & Malawi & 840 & $0-2$ & $5,1-10,6$ & z-score -0,12 & Sí \\
\hline Rivero (9) & 2018 & Argentina & 303 & $0-15$ & 33,3 & 38,9 & Sí \\
\hline Yentur (10) & 2015 & Turquía & 100 & $0-6$ & 42,5 & 16,0 & Sí \\
\hline Heimer (11) & 2015 & Ruanda & 622 & $6-18$ & $28,0-43,9$ & z-score $-1,8$ y -0,6 & Sí \\
\hline Centeno-Lima (12) & 2013 & Guinea-Bissau & 109 & $0-5$ & 29,0 & 28,4 & No \\
\hline Al-Mekhlafi (13) & 2013 & Malasia & 374 & $7-12$ & 22,2 & 24,8 & No \\
\hline Verhagen (14) & 2013 & Venezuela & 390 & $4-16$ & 20,0 & $13-84$ & Sí \\
\hline Zonta (15) & 2010 & Argentina & 178 & $1-14$ & 20,0 & 44,9 & Sí \\
\hline Maia (16) & 2009 & Brasil & 451 & $0-10$ & 21,5 & 17,5 & No \\
\hline Silva (17) & 2009 & Brasil & 405 & $0-6$ & 26,3 & 7,9 & No \\
\hline Nematian (18) & 2008 & Irán & 19209 & $8,5 \pm 1,5$ & 11,5 & $2,8-3,8$ & Sí \\
\hline Muniz-Junqueira (19) & 2002 & Brasil & 124 & $1-6$ & 30,4 & 16,0 & Sí \\
\hline Berkman (20) & 2002 & Perú & 239 & $0-2$ & 14,1 & 42,0 & Sí \\
\hline
\end{tabular}

y la baja talla (Tabla 4) $(7,20)$. La Giardia lambia causa disminución en la ingesta de los requerimientos nutricionales a nivel corporal, debido a su interrelación con las superficies de la absorción intestinal y el consumo de nutrientes por el organismo, como consecuencia de la obstrucción física a nivel de la luz intestinal y de la producción de sustancias proteolíticas, entre otras (30). Se cree que las infecciones parasitarias contribuyen a la desnutrición mediante la reducción sutil de la digestión y la absorción, la inflamación crónica y la pérdida de nutrientes (31). Se sabe que Giardia causa diarrea aguda, malabsorción de grasas, vitaminas y $\mathrm{D}$-xilosa, e intolerancia a la lactosa, especialmente entre los niños (32). Además, está bien documentado que los trofozoítos de Giardia causan un trastorno de la arquitectura vellosa normal con acortamiento de las vellosidades y focos inflamatorios en las criptas y la lámina 
propia, lo que resulta en malabsorción (31). Todos estos mecanismos contribuyen al desarrollo de la desnutrición entre las personas infectadas (33).

Hay una gran variación en los resultados de los estudios que evalúan el efecto de la infección por $H$. pylori en el crecimiento de los niños, apuntando a la presencia o ausencia de esta asociación (Tabla 5) (21, 27). El mecanismo por el cual $H$. pylori causa deterioro del crecimiento en los niños aún no se ha investigado, ya que no está claro si el compromiso del crecimiento es un efecto directo de la inflamación inducida por $H$. pylori o una consecuencia de efectos indirectos como la anorexia inducida por infección, los cambios de permeabilidad intestinal asociados con H. pylori, la malabsorción o la enfermedad diarreica (34). Actualmente no se puede apartar de las posibles causas de baja talla en niños la disfunción entérica ambiental (DEA), alteración generalizada de la estructura y función del intestino delgado y estado subclínico de inflamación intestinal, pérdida intestinal, mayor permeabilidad, disbiosis, translocación bacteriana, inflamación sistémica y malabsorción de nutrientes (35); resultado de la exposición crónica a patógenos, que involucra saneamiento y retraso del crecimiento (36). Aunque aún no se define totalmente la patogenia entre estas dos entidades, se proponen recomendaciones nutricionales con base en intervenciones tradicionales de agua, saneamiento e higiene (WASH) (36), que incluyen la reducción de la exposición a las heces y el contacto con animales a través de programas tales como agua mejorada, saneamiento e higiene; lactancia materna y diversidad dietética mejorada; probióticos y prebióticos; suplementos nutricionales, incluyendo cinc, ácidos grasos poliinsaturados y aminoácidos; agentes antiinflamatorios tales como ácido 5-aminosalicíclico; y antibióticos en el contexto de la desnutrición aguda y la infección (35).
A futuro, se piensa que las tecnologías "ómicas" (genómica, epigenómica, transcriptómica, proteómica y metabolómica) y técnicas de isótopos estables (por ejemplo, pruebas de aliento 13C) dirigidas a niños y su microbiota intestinal mejorarán la capacidad para identificar, manejar y prevenir con éxito este trastorno (35).

La duodenitis eosinofílica en este estudio estuvo presente en 1 paciente que tuvo como síntoma principal la no ganancia de peso; dato también descrito por otros autores $(37,38)$ y que aparentemente pueden responder adecuadamente al montelukast (39). Otros síntomas digestivos en niños con duodenitis eosinofílica son el dolor abdominal y la diarrea $(37,38)$. A nivel del duodeno, los cambios histopatológicos reportados en este grupo de niños son la microerosión y la fibrosis (40), sin que los biomarcadores sean pruebas diagnósticas a considerar en ellos comúnmente (41).

En este estudio, solo un niño presentó úlcera prepilórica. La enfermedad ulceropéptica en pediatría no es frecuente: se reporta entre un $0,6 \%$ y $12,8 \%$ de úlceras gástricas o duodenales $(42,43)$; sin embargo, la morbilidad puede incluir complicaciones graves, como perforación, sangrado, estenosis y obstrucción intestinal. El compromiso ponderoestatural de los niños con enfermedad acidopéptica está asociado en mayor o menor grado con la presencia de síntomas, que en edades tempranas pueden ser irritabilidad, mala alimentación, regurgitación, vómito o sangrado digestivo; y en niños mayores, dolor abdominal, flatulencias, distensión abdominal, náuseas y trastornos del sueño, entre otros (44).

Dos niños presentaron hiperplasia nodular linfoide duodenal en este estudio, 1 de ellos con H. pylori gástrico, asociación reportada por Blanco y colaboradores (45). Aunque en la edad pediátrica puede encontrarse en la mucosa del tubo

Tabla 5. H. pylori y baja talla

\begin{tabular}{lllllllll}
\multicolumn{1}{c}{ Autor } & Año & \multicolumn{1}{c}{ País } & \multicolumn{1}{c}{$\mathbf{n}$} & \multicolumn{1}{c}{ Edad (años) } & $\begin{array}{c}\text { H. pylori } \\
(\%)\end{array}$ & Falla para crecer & Asociación \\
\hline Chiu (21) & 2017 & Taiwán & 53 & $4-18$ & 29,2 & $32,0 \%$ & No \\
\hline Kocaoglu (22) & 2016 & Turquía & 200 & $7-18$ & 59,5 & ${ }^{*} 0,49 \pm 3,85$ & Sí \\
\hline Soylu (23) & 2008 & Turquía & 108 & $6,9-17,3$ & 52,8 & $12,3 \%$ & No \\
\hline Fialho (24) & 2007 & Brasil & 353 & $0,6-14$ & 55,8 & ${ }^{* *} 6,62($ IC $95 \% 1,71-25,55, p=0,006)$ & Sí \\
\hline Süoglu (25) & 2007 & Turquía & 70 & $4-16$ & 50,0 & ${ }^{* * *}-0,768 \pm 1.252$ & Sí \\
Chimonas (26) & 2006 & Estados Unidos & 650 & $7-11$ & 87,1 & $39,0 \% \pm 26,4 \%$ & No \\
\hline Richter (27) & 2001 & Alemania & 3315 & $5-7$ & 6,2 & ${ }^{* * *}-0,253 \pm 1,088$ & Sí
\end{tabular}

${ }^{*}$ Velocidad de crecimiento. ${ }^{* *}$ Odds ratio (OR). ${ }^{* * *}$ Promedio \pm DE. IC: intervalo de confianza. 
digestivo mayor cantidad de tejido linfoide que en el adulto, la existencia de duodeno nodular es un hallazgo endoscópico poco común y de significado clínico incierto, y su repercusión clínica antropométrica es poco clara. La hiperplasia linfoide se ha descrito en niños con alergia alimentaria, hipogammaglobulinemia (46), infestación por G. lamblia (47), pacientes con insuficiencia renal terminal y con enfermedad péptica secundaria a infección por H. pylori (45).

En conclusión, a pesar de que la EVD es un método poco utilizado y no bien descrito en niños con baja talla, en este reporte de casos, se logró determinar organicidad en un $80,0 \%$ de los niños a quienes se les realizó una EVDA.

\section{REFERENCIAS}

1. Becker P, Carney LN, Corkins MR, Monczka J, Smith E, Smith SE, Spear BA, White JV; Academy of Nutrition and Dietetics; American Society for Parenteral and Enteral Nutrition. Consensus statement of the Academy of Nutrition and Dietetics/American Society for Parenteral and Enteral Nutrition: indicators recommended for the identification and documentation of pediatric malnutrition (undernutrition). Nutr Clin Pract. 2015;30(1):147-61. https://doi.org/10.1177/0884533614557642

2. Larson-Nath C, Biank VF. Clinical Review of Failure to Thrive in Pediatric Patients. Pediatr Ann. 2016;45(2):e469. https://doi.org/10.3928/00904481-20160114-01

3. Homan GJ. Failure to Thrive: A Practical Guide. Am Fam Physician. 2016;94(4):295-9.

4. Bouma S. Diagnosing Pediatric Malnutrition. Nutr Clin Pract. 2017;32(1):52-67. https://doi. org $/ 10.1177 / 0884533616671861$

5. de Onis M, Onyango AW, Borghi E, Siyam A, Nishida C, Siekmann J. Development of a WHO growth reference for school-aged children and adolescents. Bull World Health Organ. 2007;85(9):660-7. https://doi.org/10.2471/ blt.07.043497

6. Thomson M, Tringali A, Dumonceau JM, Tavares M, Tabbers MM, Furlano R, Spaander M, Hassan C, Tzvinikos C, Ijsselstijn H, Viala J, Dall'Oglio L, Benninga M, Orel R, Vandenplas Y, Keil R, Romano C, Brownstone E, Hlava Š, Gerner P, Dolak W, Landi R, Huber WD, Everett S, Vecsei A, Aabakken L, Amil-Dias J, Zambelli A. Paediatric Gastrointestinal Endoscopy: European Society for Paediatric Gastroenterology Hepatology and Nutrition and European Society of Gastrointestinal Endoscopy Guidelines. J Pediatr Gastroenterol Nutr. 2017;64(1):133153. https://doi.org/10.1097/MPG.0000000000001408

7. Ihejirika OC, Nwaorgu OC, Ebirim CI, Nwokeji CM. Effects of intestinal parasitic infections on nutritional status of primary children in Imo State Nigeria. Pan Afr Med J. 2019;33:34. https://doi.org/10.11604/ pamj.2019.33.34.17099

8. Lehto KM, Fan YM, Oikarinen S, Nurminen N, Hallamaa L, Juuti R, Mangani C, Maleta K, Hyöty H, Ashorn P. Presence of Giardia lamblia in stools of six- to 18-month old asymptomatic Malawians is associated with children's growth failure. Acta Paediatr. 2019;108(10):1833-1840. https://doi.org/10.1111/apa.14832
9. Rivero MR, De Angelo C, Nuñez P, Salas M, Liang S. Intestinal parasitism and nutritional status among indigenous children from the Argentinian Atlantic Forest: Determinants of enteroparasites infections in minority populations. Acta Trop. 2018;187:248-256. https://doi. org/10.1016/j.actatropica.2018.08.015

10. Yentur Doni N, Yildiz Zeyrek F, Simsek Z, Gurses G, Sahin İ. Risk Factors and Relationship Between Intestinal Parasites and the Growth Retardation and Psychomotor Development Delays of Children in Şanlıurfa, Turkey. Turkiye Parazitol Derg. 2015;39(4):270-6. https://doi. org/10.5152/tpd.2015.3620

11. Heimer J, Staudacher O, Steiner F, Kayonga Y, Havugimana JM, Musemakweri A, Harms G, Gahutu JB, Mockenhaupt FP. Age-dependent decline and association with stunting of Giardia duodenalis infection among schoolchildren in rural Huye district, Rwanda. Acta Trop. 2015;145:17-22. https://doi.org/10.1016/j.actatropica.2015.01.011

12. Centeno-Lima S, Rosado-Marques V, Ferreira F, Rodrigues R, Indeque B, Camará I, De Sousa B, Aguiar P, Nunes B, Ferrinho P. Giardia Duodenalis e Desnutrição Crónica em Crianças Menores de Cinco Anos de uma Região Rural da Guiné-Bissau. Acta Med Port. 2013;26(6):721-4.

13. Al-Mekhlafi HM, Al-Maktari MT, Jani R, Ahmed A, Anuar TS, Moktar N, Mahdy MA, Lim YA, Mahmud R, Surin $\mathrm{J}$. Burden of Giardia duodenalis infection and its adverse effects on growth of schoolchildren in rural Malaysia. PLoS Negl Trop Dis. 2013;7(10):e2516. https://doi. org/10.1371/journal.pntd.0002516

14. Verhagen LM, Incani RN, Franco CR, Ugarte A, Cadenas Y, Sierra Ruiz CI, Hermans PW, Hoek D, Campos Ponce $\mathrm{M}$, de Waard JH, Pinelli E. High malnutrition rate in Venezuelan Yanomami compared to Warao Amerindians and Creoles: significant associations with intestinal parasites and anemia. PLoS One. 2013;8(10):e77581. https:// doi.org/10.1371/journal.pone.0077581

15. Zonta ML, Oyhenart EE, Navone GT. Nutritional status, body composition, and intestinal parasitism among the Mbyá-Guaraní communities of Misiones, Argentina. Am J Hum Biol. 2010;22(2):193-200. https://doi.org/10.1002/ ajhb.20977

16. Maia MM, Fausto MA, Vieira EL, Benetton ML, Carneiro $M$. Intestinal parasitic infection and associated risk factors, among children presenting at outpatient clinics in 
Manaus, Amazonas state, Brazil. Ann Trop Med Parasitol. 2009;103(7):583-91. https://doi.org/10.1179/0003498 09X12459740922417

17. Silva RR, da Silva CA, de Jesus Pereira CA, de Carvalho Nicolato RL, Negrão-Corrêa D, Lamounier JA, Carneiro M. Association between nutritional status, environmental and socio-economic factors and Giardia lamblia infections among children aged 6-71 months in Brazil. Trans R Soc Trop Med Hyg. 2009; 103(5):512-9. https://doi. org/10.1016/j.trstmh.2008.10.019

18. Nematian J, Gholamrezanezhad A, Nematian E. Giardiasis and other intestinal parasitic infections in relation to anthropometric indicators of malnutrition: a large, population-based survey of schoolchildren in Tehran. Ann Trop Med Parasitol. 2008;102(3):209-14. https://doi. org/10.1179/136485908X267876

19. Muniz-Junqueira MI, Queiroz EF. Relationship between protein-energy malnutrition, vitamin $\mathrm{A}$, and parasitoses in living in Brasília. Rev Soc Bras Med Trop. 2002;35(2):133 41. https://doi.org/10.1590/s0037-86822002000200002

20. Berkman DS, Lescano AG, Gilman RH, Lopez SL, Black MM. Effects of stunting, diarrhoeal disease, and parasitic infection during infancy on cognition in late childhood: a follow-up study. Lancet. 2002;359(9306):564-71. https:// doi.org/10.1016/S0140-6736(02)07744-9

21. Chiu NC, Lin CY, Chi H, Yeung CY, Ting WH, Chan WT, Jiang CB, Li ST, Lin CH, Lee HC. Helicobacter pylori infection is not associated with failure to thrive: a case control study. Ther Clin Risk Manag. 2017;13:273-278. https://doi.org/10.2147/TCRM.S123148

22. Kocaoglu C, Ozel A, Cayci M, Solak ES. Effect of longterm Helicobacter pylori infection on growth of children: a cohort study. World J Pediatr. 2016;12(2):196-201. https://doi.org/10.1007/s12519-015-0021-7

23. Soylu OB, Ozturk Y. Helicobacter pylori infection: effect on malnutrition and growth failure in dyspeptic children. Eur J Pediatr. 2008;167(5):557-62. https://doi.org/10.1007/ s00431-007-0552-6

24. Fialho AM, Braga AB, Queiroz DM, Rodrigues MN, Herbster ID, Braga LL. The association between Helicobacter pylori infection and height in children from an urban community in north-east Brazil. Ann Trop Paediatr. 2007;27(1):55-61. https://doi. org/10.1179/146532807X170510

25. Süoglu OD, Gökçe S, Saglam AT, Sökücü S, Saner G. Association of Helicobacter pylori infection with gastroduodenal disease, epidemiologic factors and iron-deficiency anemia in Turkish children undergoing endoscopy, and impact on growth. Pediatr Int. 2007;49(6):858-63. https://doi.org/10.1111/j.1442-200X.2007.02444.x

26. Chimonas MA, Baggett HC, Parkinson AJ, Muth PT, Dunaway E, Gessner BD. Asymptomatic Helicobacter pylori infection and iron deficiency are not associated with decreased growth among Alaska Native children aged 7-11 years. Helicobacter. 2006;11(3):159-67. https://doi. org/10.1111/j.1523-5378.2006.00395.x
27. Richter T, Richter T, List S, Müller DM, Deutscher J, Uhlig $\mathrm{HH}$, Krumbiegel P, Herbarth O, Gutsmuths FJ, Kiess W. Five- to 7-year-old children with Helicobacter pylori infection are smaller than Helicobacter-negative children: a cross-sectional population-based study of 3,315 children.J Pediatr Gastroenterol Nutr. 2001;33(4):472-5. https://doi. org/10.1097/00005176-200110000-00010

28. Larson-Nath CM, Goday PS. Failure to Thrive: A Prospective Study in a Pediatric Gastroenterology Clinic. J Pediatr Gastroenterol Nutr. 2016;62(6):907-13. https:// doi.org/10.1097/MPG.0000000000001099

29. El Mouzan MI, Assiri AM, Al Herbish AS, Al Sohaibani MO. Endoscopic duodenal biopsy in children. Saudi J Gastroenterol. 2006;12(1):31-3. https://doi. org/10.4103/1319-3767.27742

30. Stephenson LS, Latham MC, Ottesen EA. Malnutrition and parasitic helminth infections. Parasitology. 2000;121 Suppl:S23-38. https://doi.org/10.1017/ s0031182000006491

31. Northrop-Clewes CA, Rousham EK, Mascie-Taylor CN, Lunn PG. Anthelmintic treatment of rural Bangladeshi children: effect on host physiology, growth, and biochemical status. Am J Clin Nutr. 2001;73(1):53-60. https://doi. org/10.1093/ajcn/73.1.53

32. Gendrel D, Treluyer JM, Richard-Lenoble D. Parasitic diarrhea in normal and malnourished children. Fundam Clin Pharmacol. 2003;17(2):189-97. https://doi.org/10.1046/ j.1472-8206.2003.00169.x

33. Halliez MC, Buret AG. Extra-intestinal and long term consequences of Giardia duodenalis infections. World J Gastroenterol. 2013;19(47):8974-85. https://doi. org/10.3748/wjg.v19.i47.8974

34. Queiroz DM, Rocha AM, Crabtree JE. Unintended consequences of Helicobacter pylori infection in children in developing countries: iron deficiency, diarrhea, and growth retardation. Gut Microbes. 2013;4(6):494-504. https:// doi.org/10.4161/gmic.26277

35. Owino V, Ahmed T, Freemark M, Kelly P, Loy A, Manary M, Loechl C. Environmental Enteric Dysfunction and Growth Failure/Stunting in Global Child Health. Pediatrics. 2016;138(6):e20160641. https://doi. org/10.1542/peds.2016-0641

36. Budge S, Parker AH, Hutchings PT, Garbutt C. Environmental enteric dysfunction and child stunting. Nutr Rev. 2019;77(4):240-253. https://doi.org/10.1093/ nutrit/nuy068

37. Holguín L, Gallego-Yépes C, Toro Y, Díez-Zuluaga LS, Mopan J, Chinchilla C. Caracterización epidemiológica, clínica y diagnóstica de niños con gastroenteropatía eosinofílica. Estudio retrospectivo de tres instituciones de salud de alta complejidad. Rev Alerg Mex. 2018;65(2):148-159. https://doi.org/10.29262/ram.v65i2.354

38. Busoni VB, Lifschitz C, Christiansen S, G de Davila MT, Orsi M. Gastroenteropatía eosinofílica: una serie pediátrica. Arch Argent Pediatr. 2011;109(1):68-73. https://doi. org/10.1590/S0325-00752011000100019 
39. Friesen CA, Kearns GL, Andre L, Neustrom M, Roberts CC, Abdel-Rahman SM. Clinical efficacy and pharmacokinetics of montelukast in dyspeptic children with duodenal eosinophilia. J Pediatr Gastroenterol Nutr. 2004;38(3):343-51. https://doi.org/10.1097/00005176200403000-00021

40. Berezenko V, Bogdanova T, Krotevich M, Savenko Y, Vankhanova T. Morphological characteristics of chronic gastroduodenitis in adolescents with food hypersensitivity. Georgian Med News. 2018;(284):93-97.

41. Neilan NA, Dowling PJ, Taylor DL, Ryan P, Schurman JV, Friesen CA. Useful biomarkers in pediatric eosinophilic duodenitis and their existence: a case-control, singleblind, observational pilot study. J Pediatr Gastroenterol Nutr. 2010;50(4):377-84. https://doi.org/10.1097/ MPG.0b013e3181c2c28a

42. Akbulut UE, Emeksiz HC, Kocak FG, Livaoglu A. Diagnostic yield of esophagogastroduodenoscopy in children with chronic abdominal pain. Arch Med Sci. 2018;14(1):74-80. https://doi.org/10.5114/ aoms.2017.67675

43. Thakkar K, Chen L, Tessier ME, Gilger MA. Outcomes of children after esophagogastroduodenoscopy for chronic abdominal pain. Clin Gastroenterol Hepatol. 2014;12(6):963-9. https://doi.org/10.1016/j. cgh.2013.08.041

44. Sierra D, Wood M, Kolli S, Felipez LM. Pediatric Gastritis, Gastropathy, and Peptic Ulcer Disease. Pediatr Rev. 2018;39(11):542-549. https://doi.org/10.1542/pir.20170234

45. Blanco Rodríguez G, García Cárdenas E, Velasco Sánchez F, Bernal Redondo RM, López Facio K, Faure Fontenla A, Penchyna Grub J, Valencia Mayoral P. Hallazgos clínicopatológicos en niños con nódulos duodenales. Patologia (Mex). 2011;49(4):257-261.

46. Pernas Gómez P, Iglesias Meleiro J, García Rodríguez C, Martiñón Sánchez F. Dos observaciones de hiperplasia nodular linfoide intestinal asociadas a déficits inmunitarios. An Pediatr. 2011;75(1):72-3. https://doi.org/10.1016/j. anpedi.2010.12.008

47. Gonul CD, Bilge C, Gazi KA, Filiz K. Duodenal nodularity in children: a clinical and pathologic study of 17 cases. Indian J Pathol Microbiol. 2011;54(2):312-7. https://doi. org/10.4103/0377-4929.81611 